\title{
API et enseignement de la prononciation du français en Chine : médiation ou interférence?
}

\author{
Junkai $\mathrm{LI}^{1}$, Yi $\mathrm{YIN}^{2}$ et Zhihong $\mathrm{PU}^{1, *}$ \\ ${ }^{1}$ CEF-FLE, Université Sun Yat-sen, 135 Xingangxi Road, 510275 Guangzhou, Chine \\ ${ }^{2}$ UMR7018-LPP, Université Sorbonne Nouvelle Paris 3, 19 rue des Bernardins, 75005 Paris, France
}

\begin{abstract}
Résumé. La prononciation constitue une des difficultés majeures pour les apprenants chinois. En Chine, l'alphabet phonétique international (API) est privilégié dans l'enseignement de la prononciation du français. S'agit-il d'une médiation qui facilite l'apprentissage ou d'une interférence qui en réduit l'efficacité étant donné que le français est une langue alphabétique dont l'orthographe indique déjà la prononciation dans une large mesure ? Cet article vise à évaluer le rôle de l'API dans l'enseignement de la prononciation du français chez les étudiants chinois à travers une comparaison entre la méthode syllabique et celle par l'API. Les résultats des analyses de corrélation sous SPSS suggèrent que les étudiants formés avec la méthode syllabique maîtrisent mieux la prononciation du français par rapport à ceux formés avec la méthode API.
\end{abstract}

\begin{abstract}
IPA and French pronunciation teaching in China : mediation or interference? Pronunciation is one of the major difficulties for Chinese students of French when they start learning it, and the International Phonetic Alphabet (IPA) is largely adopted in French pronunciation teaching in China. Is it a mediation that facilitates learning or an interference that reduces its effectiveness given that French is an alphabetic language whose orthography indicates already the pronunciation to a large extent? This article aims to evaluate the role of the IPA in French pronunciation teaching in Chinese context through a contrastive study between the phonics method and the IPA one. The results of the SPSS correlation analysis suggest that the students taught with the phonics method demonstrate a better level of pronunciation than those who trained with the IPA method.
\end{abstract}

\footnotetext{
*Corresponding author : puzhihong@hotmail.com
} 


\section{Introduction}

Les étudiants de spécialité universitaire «langue et littérature françaises » en Chine sont majoritairement des débutants. L'apprentissage de la prononciation est pour eux le premier pas dans la découverte de la langue de Molière. Cependant, à cause des particularités qui s'inscrivent dans les caractéristiques phonétiques et phonologiques du français, le rôle de l'enseignement de la prononciation du français s'avère plus marqué et incontournable par rapport à celui d'autres langues comme l'allemand, le japonais ou l'espagnol. Ainsi, plus de défis se posent aux enseignants de français langue étrangère (ci-après FLE) en Chine. Au cours du $20^{\mathrm{e}}$ siècle, cependant, la prononciation a souvent fait figure d'orpheline de la didactique, hormis dans certaines approches méthodologiques (Detey, Fontan \& Pellegrini, 2016 : 16). Il est à noter aussi que les étudiants spécialisés en français possèdent souvent un niveau assez élevé en expression et compréhension écrites alors que leur compétence de compréhension orale, et surtout celle d'expression orale, restent relativement restreintes. Certains étudiants qui apprennent le français en tant que $2^{\mathrm{e}}$ langue étrangère ne réussissent même pas à bien reproduire oralement un mot entier ou une phrase complète au bout de deux ans d'apprentissage, sans parler de l'accentuation, de l'intonation, ou de la qualité et l'efficacité de la communication.

L'une des difficultés de la prononciation pour nos apprenants consiste à associer la graphie française à sa phonie sans que l'on recoure à la transcription phonétique. Pour mieux maîtriser l'oralité du français, un autre système d'annotation est utilisé par les apprenants. Certains annotent les graphies par l'alphabet phonétique international (API) alors que d'autres font appel à l'anglais, aux caractères chinois, au pinyin (romanisation des caractères chinois) ou à son propre système de transcription. L'API étant une transcription scientifique qui indique d'une façon plutôt exacte la prononciation d'un mot alors que dans d'autres moyens de transcription, certains phonèmes du français n'existent pas du tout comme en anglais et en chinois. Effectivement, le but de la création de l'API est destiné aux chercheurs en linguistique ou en didactologie, mais pas aux élèves. Ainsi, l'API est trop compliqué de sorte qu'il n'est pas opportun de l'appliquer chez les débutants (Cheng \& Fan, 2011 : 79).

En effet, pour des raisons historiques et politiques, la dépendance de l'API en Chine hérite de la tradition pédagogique de l'ex-URSS selon laquelle l'utilisation de l'API est privilégiée dans l'enseignement des langues. Ainsi, l'enseignement du FLE en Chine s'inscrit conventionnellement dans cette méthode comme s'il était universel et légitime dans le monde entier de transcrire phonétiquement une écriture (ibid.: 80). Jusqu'à aujourd'hui, l'API est toujours considéré comme un outil très important dans l'enseignement de la prononciation des langues étrangères en Chine et nous ne pouvons pas nous en passer. Etant donné que la pédagogie "lettre $\rightarrow$ transcription $\rightarrow$ prononciation » encadre l'enseignement / apprentissage de la prononciation des langues étrangères en Chine, une partie de nos étudiants ont des difficultés à oraliser des mots français sans le recours à la transcription phonétique. Certes, l'API nous permet de connaître la prononciation exacte d'une graphie alors qu'il dissocie la correspondance graphie-phonie intrinsèque de cette langue alphabétique. Gougenheim (1935) prétend que 70\% de l'orthographe en français connaît une correspondance biunivoque avec sa réalisation phonétique. Ainsi, il paraît plus facile et efficace d'établir une relation entre les lettres et leur prononciation au cours de l'apprentissage du français que de recourir à un intermédiaire qui n'a rien à voir avec la forme écrite de la langue. De ce fait, nous nous interrogeons donc sur le rôle de l'API dans l'enseignement de la prononciation du français en présentant ses apports et ses limites en contexte universitaire chinois. Dans le présent travail, nous retraçons d'abord l'enjeu de l'enseignement de la prononciation du français en Chine. Nous évoquons ensuite les avantages et inconvénients de l'API en contexte chinois ainsi que quelques réflexions sur la 
relation graphie-phonie dans l'apprentissage de la prononciation du FLE. Enfin, à partir d'une étude empirique, nous nous permettons de tenter une réflexion sur de nouvelles orientations de l'enseignement de la prononciation du français qui peuvent mieux s'adapter au profil des apprenants chinois.

\section{Place de la prononciation dans l'enseignement du FLE}

L'apprentissage d'une langue étrangère implique la maîtrise de nombreuses compétences, celle de la prononciation n'étant pas la moindre. Cependant, l'apprentissage de la prononciation est parfois négligé ou marginalisé en Occident du fait de la typologie linguistique assez proche entre les langues maternelles des apprenants européens. Grâce à la forte intercompréhension linguistique, les apprenants européens partageant les langues premières proches ou apparentées semblent avoir un apprentissage / acquisition des langues secondes plus aisé par rapport à des locuteurs des langues éloignées (cf. Robert, 2004 : 500501). De ce fait, en comparaison des étudiants asiatiques, les apprenants européens oralisent plus facilement ce qu'ils voient ou ce qu'ils entendent dès le premier contact avec une autre langue européenne ayant comme écriture commune l'alphabet romain. Rappelons qu'en Europe, le Cadre européen commun de référence pour les langues (Conseil de l'Europe, 2001 : 92) a défini six niveaux de compétence phonologique :

Tableau 1. Six niveaux de compétence phonologique définis par le CECRL

\begin{tabular}{|l|l|}
\cline { 2 - 3 } & \multicolumn{1}{|c|}{ MAITRISE DU SYSTÈME PHONOLOGIQUE } \\
\hline C2 & Comme C1 \\
\hline C1 & Peut varier l'intonation et placer l'accent phrastique correctement afin d'exprimer de fines nuances de sens. \\
\hline B2 & A acquis une prononciation et une intonation claires et naturelles. \\
\hline B1 & $\begin{array}{l}\text { La prononciation est clairement intelligible même si un accent étranger est quelquefois perceptible et si des erreurs de } \\
\text { prononciation proviennent occasionnellement. }\end{array}$ \\
\hline A2 & $\begin{array}{l}\text { La prononciation est en général suffisamment claire pour être comprise malgré un net accent étranger mais l'interlocuteur } \\
\text { devra parfois faire répéter. }\end{array}$ \\
\hline A1 & $\begin{array}{l}\text { La prononciation d'un répertoire très limité d'expressions et de mots mémorisés est compréhensible avec quelque effort } \\
\text { pour un locuteur natif habitué aux locuteurs du groupe linguistique de l'apprenant/utilisateur. }\end{array}$ \\
\hline
\end{tabular}

Néanmoins, nous remarquons dans ce tableau l'importance attachée à un aspect communicationnel : la réception et la production, de préférence sous forme d'interactions orales. Cela implique nécessairement, dès les premiers niveaux d'apprentissage, l'acquisition de la prononciation de la langue cible chez l'apprenant. Toutefois, l'attention accordée à la phonétique et à la phonologie a toujours été bien trop réduite. Or, bien que ce tableau définisse les compétences demandées pour chaque niveau, les consignes sont loin d'être précises. La compétence de communication semble être accentuée au détriment de la prononciation du point de vue phonético-phonologique. D'ailleurs, l'élaboration de ces critères est basée sur le profil des apprenants européens au lieu de celui des apprenants asiatiques, leurs particularités en matière de la culture d'apprentissage et la particularité de leur langue maternelle n'ont pas été naturellement prises en considération.

L'enseignement de la prononciation étant en évolution constante depuis le $20^{\mathrm{e}}$ siècle, et particulièrement depuis ces dernières décennies, nous vivons ainsi des transformations très importantes, surtout en raison de la vulgarisation des ressources numériques et des réseaux sociaux. De nouvelles approches ayant pour objectif la communication émergent, et les enseignants et apprenants doivent tous s'y adapter. Un des courants didactiques qui introduit l'enseignement de la prononciation s'intitule « la méthode directe », dans laquelle 
l'imitation est préconisée. Cette méthode s'inspire de l'acquisition de la prononciation de la langue maternelle chez l'enfant. Le développement de la phonétique expérimentale engendre la méthode structuro-globale audiovisuelle (SGAV) et l'approche analytique. C'est dans cette période que la notion de « crible phonologique » de Troubetzkoy (1986) a été reprise. Des approches pédagogiques engageant le corps et la voix dans l'apprentissage de la prononciation sont abordées et utilisées par les enseignants. La chanson est aussi une médiation facilitant l'appropriation à la suggestopédie. De façon idéale, la prononciation s'apprend avec l'imitation des natifs, et l'oral doit l'emporter sur l'écrit. Cependant, à cause des contraintes institutionnelles dans un cours de langue à l'université en Chine, l'écrit est considéré comme plus important par rapport à l'oral et gagne en priorité sur l'enseignement / apprentissage du FLE qui vise essentiellement à préparer les étudiants à la réussite des examens universitaires et nationaux. De ce fait, dans un contexte exolingue, il nous convient de développer davantage chez les apprenants chinois la "compétence orthoépique » (Conseil de l'Europe, 2001 : 92), c'est-à-dire la capacité de produire une prononciation correcte à partir de la forme écrite. Cette relation entre l'oral et l'écrit implique la maîtrise des règles orthographiques et des conventions qui y sont mises en œuvre pour représenter la prononciation.

Certes, l'oral, ou plus précisément la prononciation dans notre cas, est fondamentale pour acquérir une compétence de communication du fait qu'elle conditionne dès le début l'apprentissage de la compréhension et l'expression orale. Comme soulignent Cuq et Gruca :

«Savoir reproduire les phonèmes dont certains sont spécifiques au français, s'imprégner de l'enchaînement syllabique, produire une suite de sons qui fassent sens et qui correspondent à une pensée, [...] sont autant d'éléments difficiles à acquérir et à gérer en situation de parole et qui constituent, cependant, le b-a ba d'un seuil de communication.» (2005 : 179)

Ceci dit, la prononciation d'un locuteur affecte l'intelligibilité de la communication. Or, sous l'influence de cette nouvelle tendance, la didactique de l'expression orale, ainsi que la prononciation des langues étrangères, sont moins touchées. Les raisons sont diverses: Iivonen \& Nevalainen (1998: 15) confirment que les enseignants privilégient d'autres caractéristiques que le côté phonétique ; ils croient que la conscience phonétique fait partie de la conscience linguistique dont la conscience des fautes de prononciation (ibid. : 20). D'ailleurs, Lauret (2007: 15) estime que la prononciation est « une compétence physique qui recouvre à la fois la perception et la production » de l'apprenant. Ainsi, l'enseignement / apprentissage demande non seulement la compétence pédagogique de l'enseignant, mais aussi la capacité auditive, la personnalité, la motivation etc. de l'apprenant. De cette manière, la didactique de l'oral a certes pour objectif non seulement de développer la compétence de communication chez l'apprenant, mais aussi de lui apprendre à prononcer correctement la langue cible. Etant donné qu'en Chine, l'enseignement / apprentissage des langues étrangères s'appuie largement sur l'écrit, même en ce qui concerne la prononciation, l'enseignement commence très souvent par la présentation de la transcription écrite des sons pour introduire les règles de prononciation. Cet accent mis sur l'écrit est a priori lié à la tradition d'apprentissage chinoise, et le rapport entre l'écrit et l'oral s'avère subtil dans l'enseignement. D'une façon plus générale, la linguistique de l'écrit considère que l'on ne peut aborder le domaine de la lecture ou de l'écriture sans tenir compte du fonctionnement graphique d'une langue. Sa contribution se situe par conséquent dans le prolongement de ce qui vient d'être dit, mais avec des avancées théoriques qui permettent désormais de prendre une meilleure mesure de ce que les écritures ont en commun, mais également de ce qui les différencie (Jaffré, 2003 : 41).

Nous voyons ainsi bien l'importance de l'enseignement / apprentissage de la prononciation dans le parcours d'apprentissage du français en tant que langue étrangère en Chine. L'attention doit être accordée donc à la mise en relation entre l'écrit et l'oral pour 
nos apprenants. Une pédagogie plus appropriée reste à concrétiser afin d'approfondir cette compétence chez l'apprenant.

\section{API : médiation ou interférence ?}

Comme évoqué ci-dessus, à partir de la méthode analytique, la phonétique expérimentale et la linguistique structurale interviennent dans l'enseignement / apprentissage de la prononciation des langues étrangères, et le rôle de l'API dans la pratique d'enseignement en est témoin. Développé par des phonéticiens français et britanniques sous la direction de l'Association phonétique internationale, l'API ayant vu le jour pour la première fois en 1888 , sa dernière version a été publiée en 2015. Cet alphabet phonétique repose sur le principe biunivoque que chaque son correspond à un seul symbole et vice versa. Il offre un cadre précis qui a subi l'épreuve du temps et démontré son utilité dans la description des langues et même dans leur transcription orthographique (Durand, $2005: 56$ ).

Une trentaine de symboles phonétiques au total représentent le système phonologique français, et ils sont un outil pratique pour montrer la structure phonologique du français aux apprenants. Ces symboles se réalisent dans les grandes catégories articulatoires, telles que, la sonorité, le mode d'articulation, le lieu d'articulation, le trait oral / nasal, etc. Bien évidemment, l'API a des avantages qui attirent les enseignants des langues étrangères. Tout d'abord, la simplicité et la facilité de l'API proposent aux enseignants une référence fiable qui rend la pratique d'enseignement plus efficace et plus directive car les tableaux de l'API se fondent sur des principes d'exactitude qui facilitent l'enseignement à condition que les apprenants apprennent les catégories articulatoires par cœur. Par ailleurs, comme l'API se présente sous forme de tableaux et de trapèzes vocaliques, la visualisation des traits acoustiques permet d'aider les apprenants à mémoriser ces caractéristiques. Ce rôle médiateur est confirmé dans la préface de Principles of the International Phonetic Association (1949) :

"The alphabet of the IPA is designed primarily to meet practical linguistic needs, such as putting on record the phonetic or phonemic structure of languages, furnishing learners of foreign languages with phonetic transcriptions to assist them in acquiring the pronunciation, and working out Romanic orthographies for languages written in other systems or for languages hitherto unwritten. "

Cependant, nous ne pouvons pas négliger ses limites. Tout d'abord, le manque d'input oral structuré intervient dans l'apprentissage de la prononciation via l'API. Krashen (1982) propose une hypothèse d'input dans laquelle l'accent est mis sur l'input, considéré comme la condition préliminaire de l'acquisition d'une langue. L'apprentissage ne peut pas être abouti sans exercer de l'input compréhensible assez suffisant. Mais généralement, dans un cours de langue étrangère en Chine, l'apprenant est forcé de produire de l'output, à cause du temps limité, avant de se tenir prêt. Dans ce cas-là, on ne peut apprendre l'API sans avoir appris auparavant le système phonético-phonologique de la langue cible. Il insiste ainsi sur l'efficacité et l'intelligibilité de l'input, qui à son avis sont deux critères très importants. En outre, comme dans la citation précédente, l'API est destiné a priori aux besoins des recherches linguistiques. Il est pratique pour les chercheurs, alors que pour les apprenants des langues étrangères, il est trop compliqué de les utiliser directement (cf. Cheng \& Fan, 2011 : 79) à cause de ses signes et diacritiques sophistiqués renvoyant aux traits articulatoires subtils. Par conséquent, si l'on veut bien l'employer, il faut tout de même passer plus de temps à enseigner les tableaux de l'API avant d'enseigner directement le langage. Il s'agit en quelque sorte d'un gaspillage de temps étant donné qu'il n'y a que 26 lettres en français alors qu'en API, les phonèmes français s'inscrivent dans 36 symboles. 
Il s'ensuit une interférence qui entrave l'apprentissage de la prononciation du français. Force est de constater chez nos apprenants la confusion au niveau des lettres et des signes de l'API en français. Par exemple, le signe « u » représente à la fois le son qui s'écrit <ou> et la lettre qui se prononce [y]; de la même manière, le signe « $\mathrm{y}$ » renvoie à la fois le son qui s'écrit $<u>$ et la lettre qui se prononce [i]. Ainsi, force est d'entendre [tu] pour « tu » et [biro] pour «bureau » chez les étudiants chinois formés avec la méthode API. D’ailleurs, le recours à l'API développe une certaine méfiance et de l'hésitation face aux lettres. Par exemple, la lettre $<\mathrm{a}>$ en français correspond toujours à la réalisation phonétique [a], mais les débutants n'osent prononcer cette lettre qu'en regardant la transcription phonétique entre crochets (e.g. mal [mal]). Cette pédagogie accroît sans aucun doute la difficulté et l'intensité de l'apprentissage de la prononciation du français.

Dans le contexte d'apprentissage en Chine, l'API est confronté à une deuxième interférence : le pinyin. La langue chinoise s'écrit en caractères (sinogrammes). Il s'agit d'un système logogrammique dont l'écriture n'indique pas, dans la majorité des cas, la prononciation mais le sens du mot. Afin de mieux apprendre aux enfants chinois (aux apprenants étrangers également) à lire les sinogrammes et à faciliter l'entrée et le traitement du chinois à l'ordinateur, la romanisation standardisée Projet de transcription phonétique des caractères chinois a été conçu en 1958 (dernière révision en 2012). Dès lors, les Chinois sont devenus plutôt familiers avec les 26 lettres que partagent les langues européennes, bien que la représentation phonétique de chaque lettre en pinyin ne corresponde pas toujours à celle des langues occidentales. En ce sens, pour les apprenants sinophones, le recours à la transcription phonétique de leur langue maternelle devient naturel et légitime. De ce fait, l'interférence entre l'API et le pinyin méritent toute une discussion plus approfondie. Comme les lettres du pinyin sont identiques aux 26 lettres du français, des confusions en matière de symboles sont constatées quand l'apprenant transcrit une phonie française en pinyin, ayant une représentation phonétique inéquivalente par rapport à cette première. Par exemple, en pinyin, les signes $<\mathrm{b}, \mathrm{d}, \mathrm{g}>$ indiquent en réalité les consonnes non-voisées comme $<\mathrm{p}, \mathrm{t}, \mathrm{k}>$ en API. Il en résulte que les apprenants chinois montrent une mauvaise distinction au niveau de l'opposition voisé / non-voisé des occlusives en français.

Quand on parle de l'enseignement / apprentissage de la prononciation du français en contexte universitaire chinois, les incidences de la langue anglaise ne peuvent être négligées. Ayant commencé l'apprentissage de l'anglais depuis leurs études primaires, certains phonèmes anglais affectent inconsciemment l'articulation de certains phonèmes français lorsque les étudiants chinois apprennent la prononciation du français. La voilà la troisième interférence, par exemple : le symbole «e »se réfère à une voyelle mi-fermée et non arrondie en API comme en français (e.g. été [ete]). Mais pourtant, ce symbole signifie une voyelle mi-ouverte antérieure en anglais britannique sous le système D.J. (e.g. bed [bed], [bed] en API) et une diphtongue en anglais américain sous la transcription $K$. K. (e.g. made [med], [merd] en API). Ces deux systèmes d'alphabets phonétiques sont utilisés parallèlement en Chine. Donc, une confusion d'interprétation d'un même symbole se produit entre plusieurs langues. La correspondance graphie-phonie n'est plus biunivoque. Dans ce cas-là, des erreurs d'articulation seraient plus fréquentes et l'efficacité moindre. Voici un tableau récapitulant l'exemple de l'interférence sémiographique entre l'API, le français, le pinyin et l'anglais :

Tableau 2. Représentations du signe « e »

\begin{tabular}{|l|c|}
\hline API & {$[\mathrm{e}]$} \\
\hline français (alphabet phonétique) & {$[\mathrm{e}]$} \\
\hline français (lettre) & {$[ə]$} \\
\hline
\end{tabular}




\begin{tabular}{|l|c|}
\hline pinyin & {$[ə]$} \\
\hline anglais (lettre) & {$[\mathrm{i}]$} \\
\hline anglais britannique & {$[\varepsilon]$} \\
\hline anglais américain & {$[\mathrm{ei}]$} \\
\hline
\end{tabular}

\section{Méthode syllabique : interface morpho-phonologique}

L'apprentissage de la prononciation implique la maîtrise du système phonologique de cette langue. Etant donné que l'enseignement de la prononciation en Chine dépend largement des supports écrits, il nous paraît important de sensibiliser les apprenants à la conscience phonogrammique dans l'enseignement du français. La conscience phonogrammique est étroitement liée à la correspondance entre les unités phonémiques et les unités graphiques, c'est-à-dire le rapport graphème-phonème. Les langues indo-européennes dont le français reposent sur un système d'écriture alphabétique mettant en correspondance des unités graphiques, les graphèmes, avec les unités de seconde articulation de la langue orale que sont les phonèmes (cf. Nicot-Guillorel, 2012 : 98). En admettant cette relation étroite, Kalmbach (2011) et Pierret (1985) s'accordent sur le fait que l'orthographe pose un véritable problème aux apprenants $\mathrm{du}$ français non-natifs. Si nous enseignons la prononciation par l'API sans tenir compte de la liaison entre l'écrit et l'oral, nous risquons de travailler davantage sur l'orthographe ultérieurement.

De ce fait, nous essayons de recourir à l'interface morphologie-phonologie pour mieux guider l'enseignement de la prononciation du FLE en Chine. La correspondance entre la structure morphologique et la structure orthographique de la langue y joue un rôle important. Il convient donc de rappeler d'abord la notion du «degré de transparence orthographique ». La relation entre graphie et phonie diffère d'une langue à l'autre : certaines langues, comme le croate ou l'espagnol, sont très transparentes en ce qui concerne les relations entre graphèmes et phonèmes ainsi qu'entre phonèmes et graphèmes ( $\mathrm{cf}$. Ziegler \& Montant, 2005 : 150) :

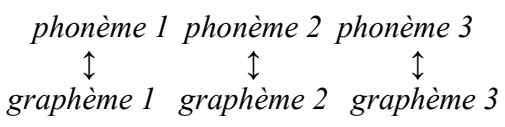

Fig. 1. Orthographe doublement transparente

Par exemple, le mot en espagnol «lentamente»se prononce [lentamente], cette transparence orthographique permet d'oraliser facilement la quasi-totalité des mots sans aucun recours à l'API. D'autres langues, comme l'anglais, sont doublement opaques, à la fois entre phonèmes et graphèmes mais aussi entre graphèmes et phonèmes (ibid.) :

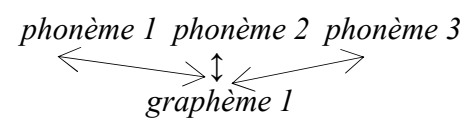

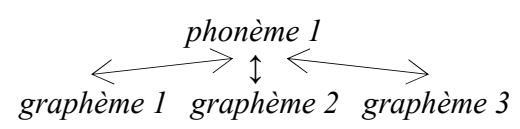

Fig. 2. Orthographe doublement opaque

Prenons comme exemple la graphie en anglais «-ough» $\left(\right.$ tough $\left[\mathrm{t}^{\mathrm{h}} \Lambda \mathrm{f}\right]$, though [ðov], through $[\theta \mathrm{ru}])$ et la phonie $[\Lambda]$ qui s'écrit diversement (cut, tough, love, blood). Dans ce cas-là, l'API s'avère une solution pertinente pour faciliter la maîtrise de la prononciation anglaise. Il existe des langues, entre autre, comme le français, qui présentent une régularité 
importante entre graphèmes et phonèmes mais paraissent relativement faibles entre phonèmes et graphèmes, certains phonèmes pouvant s'écrire de différentes façons (ibid.) :

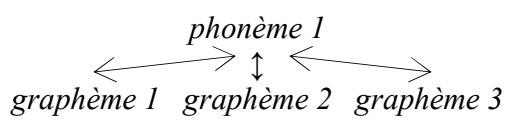

Fig. 3. Orthographe graphème-phonème transparente

En français standard (à l'exception d'un nombre limité de cas à part), le phonème $[\varepsilon]$ peut se transcrire par plusieurs graphèmes : $<\mathrm{e}>,<\hat{\mathrm{e}}\rangle,<\mathrm{ai}>$ ou $\langle\mathrm{ei}\rangle$, alors que le graphème $<$ eau $>$ ne représente que le phonème [o]. Ceci dit, si nous enseignons par l'API, soit à partir des phonèmes, il nous faut enseigner "une à plusieurs » représentations : e.g. le son [o] représente à la fois $<0>,<\mathrm{au}\rangle,<\mathrm{eau}\rangle$, et pour le phonème $[\varepsilon]$ c'est encore plus de travail. $A$ contrario, si nous partons des graphèmes, il s'agit de la relation " un à un » : e.g. la graphie $<\mathrm{ei}>$ correspond toujours à la phonie $[\varepsilon]$.
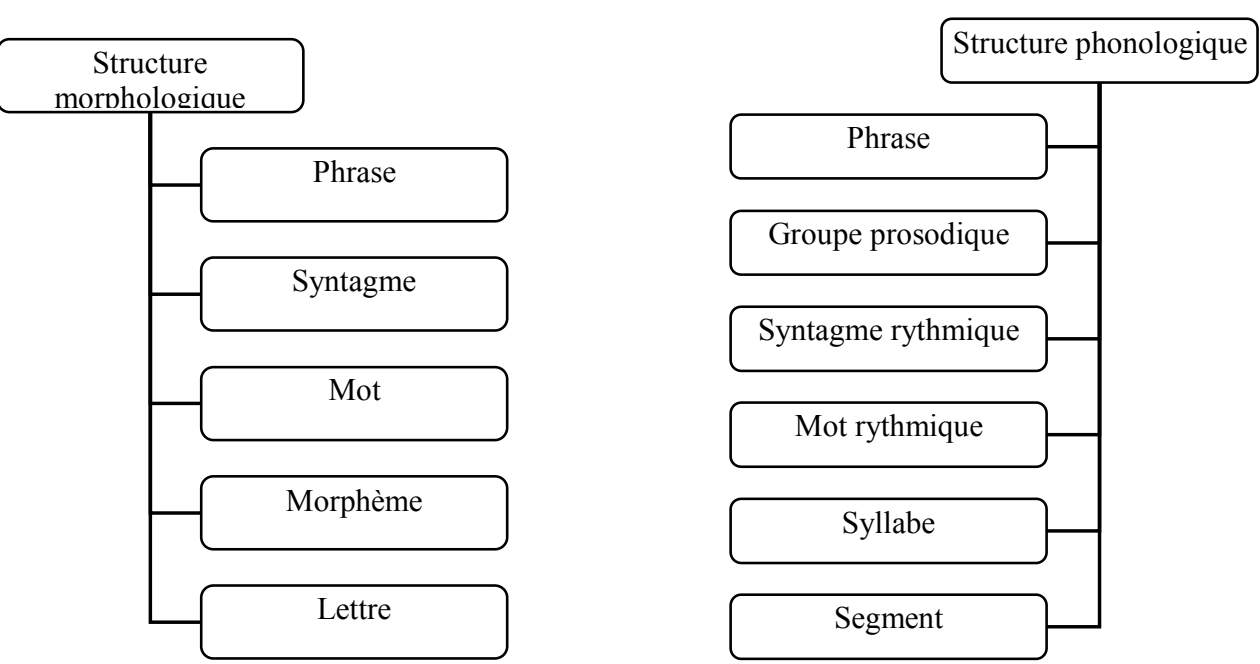

Fig. 4. Correspondance entre structures morphologiques et phonologiques (cf. Ma \& Zhao, 2017)

Le codage entre les systèmes morphologiques et phonologiques est analogue (voir Figure 4), comme les lettres se projettent sur les segments. Cette projection constitue le fondement de l'approche syllabique : apprendre à prononcer est le processus d'établir le transcodage phonographique. L'orthographe française encode à la fois la structure phonologique et morphologique des mots à l'aide de signes graphiques spécifiques (Colé \& Fayol, 2000 : 162-163). La méthode syllabique consiste de ce fait à aider les apprenants à lier le codage morphologique et le codage phonologique, c'est-à-dire à prendre conscience que la parole peut se segmenter en unités (segment, syllabe etc.) afin de saisir progressivement, d'une part, le fonctionnement des associations entre la morphologie et la phonologie et, d'autre part, le principe de leur coarticulation (fusion phonémique). Il s'agit d'un travail métalinguistique difficile reposant sur le développement progressif de la conscience phonémique jusqu'au maniement délibéré des phonèmes de la langue (cf. NicotGuillorel, 2012 : 98). Cette méthode étant largement utilisée dans l'apprentissage de la lecture de la langue maternelle en Europe, ses effets restent à explorer au niveau de l'apprentissage de la prononciation des langues étrangères, notamment en Chine. Heureusement, nous constatons des travaux (Woore, 2011) qui montre que l'enseignement orthophonique par la méthode syllabique contribue à améliorer la prononciation et la 
fluidité des apprenants du français comme langue seconde. Autrement dit, la méthode syllabique aide à réduire la difficulté d'apprentissage de la lecture (voir Figure 5.) :

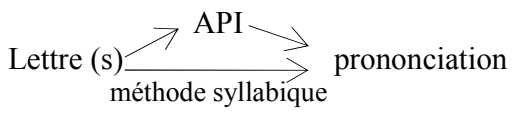

Fig. 5. Parcours entre API et méthode syllabique

Dans l'enseignement de la prononciation par la méthode syllabique, nous mettons particulièrement en relief le rôle primordial joué par certaines unités graphiques dans la perception des supports pédagogiques écrits. Ces unités se situent à l'interface entre les codes morphologiques et les codes phonologiques, et établissent des ponts fonctionnels entre ces deux systèmes de codage, constituant ainsi les éléments de base du système de lecture, soit, pour prononcer la langue apprise. En français moderne, il y a près de 130 graphèmes pour une trentaine de phonèmes et Catach (2011) évoque la notion d'« archigraphème » sur cinq critères : degré de fréquence de l'unité, degré de cohésion et de stabilité, degré de signifiance ou de pertinence phonologique, degré de rentabilité linguistique et degré de créativité linguistique :

"Parmi les quelque 130 unités que compte, au bas mot, notre écriture [...]. Parmi eux, 45 graphèmes différents présentent à leur tour un plus haut degré de fréquence, de stabilité et de pertinence. Ils peuvent être considérés comme les graphèmes de base du français, suffisants pour couvrir les besoins de communication immédiate [...] français débutant en tant que code de communication minimal ». (Catach, $2011: 60$ )

Il s'agit à cet effet de mettre l'accent sur l'apprentissage de ces phonogrammes les plus fréquentes et les plus stables, noyau de signes fonctionnels de la langue française, dans l'enseignement de la prononciation pour les débutants. Cette certitude de la stabilité phonogrammique, avec plus de $80 \%$ de correspondance biunivoque (cf. Ibid. : 64), permet d'hiérarchiser des difficultés dans l'enseignement / apprentissage de la prononciation du français si nous adoptons la méthode syllabique. Les études de Snider (1997) ont d'ailleurs prouvé que la conscience phonogrammique avait une corrélation directe avec la compétence de lecture au fur et à mesure de l'apprentissage. Elle constitue une base pour que les étudiants perfectionnent la production ainsi que la compréhension orales dans la langue cible. De ce fait, nous avons abouti à l'introduction de la méthode syllabique dans l'enseignement de la prononciation du français en Chine. Afin de mieux évaluer l'utilité de cette approche, une étude empirique contrastive menée auprès d'étudiants chinois se présente dans la partie suivante.

\section{Etude empirique comparative}

Nous avons effectué une étude empirique en vue de comparer ces deux approches d'enseignement de la prononciation du français chez les étudiants chinois à l'université. Par rapport à la méthode API, qui présente toujours la transcription par l'alphabet phonétique puis procède à lecture de ce dernier, la méthode syllabique relie directement le graphème au phonème. Pour ce faire, nous avons mis en œuvre ces deux approches dans deux classes parallèles d'une même université, l'une comme groupe expérimental et l'autre comme groupe contrôle. Le nombre d'étudiants de ces deux classes était identique et aucun étudiant n'avait de connaissance préalable du français. L'expérience s'est déroulée sur un semestre, suivi de quatre évaluations orales formatives concernant la qualité de la prononciation. Les examens ont été notés sur 100, et les notes, moyennes et écarts-types ont été analysées sous 
le logiciel SPSS en vue d'examiner la signification statistique des différences entre les résultats des deux approches à l'issue de l'enseignement.

\subsection{Sujets de l'expérience}

Le groupe expérimental compte 42 étudiants et le groupe contrôle 40, issus de la première année universitaire. Sont identiques entre les deux groupes: le rapport des sexes, la moyenne d'âge, le niveau d'études, ainsi que la connaissance du français. Il n'existe pas de différence initiale évidente entre les deux groupes au niveau de la signification statistique.

\subsection{Corpus et protocole de l'expérience}

Les deux groupes ont eu le même manuel et le même enseignant. La seule différence se trouvait dans le processus de l'enseignement de la prononciation. Le groupe contrôle suivait l'approche traditionnelle: "phonème (représenté sous forme de l'API) $\rightarrow$ transcription phonétique $\rightarrow$ lecture des transcriptions ». Le groupe expérimental s'exposait à la méthode syllabique : «lettre ou combinaison de lettres $\rightarrow$ syllabe $\rightarrow$ mot ». L'enseignement de la prononciation a duré trois mois et a été suivi de quatre tests de prononciation afin d'évaluer et de comparer les effets de ces deux approches d'enseignement.

Le premier test était composé d'une dictée de 25 mots et d'une lecture d'autant de mots. Les mots étaient issus du manuel et couvraient toutes les règles de base de prononciation du français standard y compris les oppositions voisés / non-voisés, voyelles arrondies / nonarrondies, orales / nasales, etc. Le deuxième test comprenait une dictée et une lecture de 10 groupes de mots comprenant des règles de prononciation comme l'élision, l'enchaînement et la liaison (e.g. un homme / des étudiantes / l'hôtel, elle aime / nous avons / ils habitent, etc.). En vue de mieux justifier la différence des deux approches, nous avons réalisé le troisième test (dictée et lecture de 5 phrases complètes) qui touche la prononciation au niveau suprasegmental comme les groupes rythmiques, l'accentuation et l'intonation, et le quatrième test (lecture d'un dialogue et jeu de rôle) qui consiste à simuler la prononciation dans une situation de communication quotidienne.

Tous les tests ont été notés sur 100 et nous avons comparé les notes des étudiants des deux groupes à l'aide d'une analyse statistique du logiciel SPSS. Ainsi, les moyennes des notes, les écarts-types, le test $t$, et le test one-way ANOVA ont été réalisés.

\subsection{Résultats et discussion}

Tableau 3. Analyse du premier test.

\begin{tabular}{llrrrrr}
\hline & Groupe & Nombre & Moyenne & Ecart-type & \multicolumn{1}{c}{$t$} & \multicolumn{2}{c}{$P$} \\
\hline \multirow{2}{*}{ Test 1} & expérimental & 42 & 84.12 & 10.381 & 3.159 & .002 \\
& contrôle & 40 & 76.40 & 11.729 & & \\
\hline
\end{tabular}

Selon nos analyses du test 1 , la valeur-p est inférieure au seuil de 0.05 , ce qui indique que la différence entre les deux groupes est statistiquement significative. En effet, les étudiants formés avec la méthode syllabique ont eu des notes plus élevées que ceux formés avec la méthode API. Nous remarquons chez ces derniers une confusion des signe $<\mathrm{y}>$ et $<\mathrm{u}>$ : la prononciation de la lettre $<\mathrm{y}>$ dans un mot (e.g. « cycle » prononcé comme [sykl]) et celle de l'alphabet phonétique [y] (e.g. « sur » prononcé comme [sur]).

Tableau 4. Analyse du deuxième test.

\begin{tabular}{cccccc}
\hline Groupe & Nombre & Moyenne & Ecart-type & $t$ & $P$ \\
\hline
\end{tabular}




\begin{tabular}{lllllll}
\hline Test 2 & expérimental & 42 & 81.14 & 12.007 & 2.176 & .033 \\
& contrôle & 40 & 75.50 & 11.453 & & \\
\hline
\end{tabular}

La valeur- $p$ inférieure à 0.05 signifie également que les étudiants de la méthode syllabique ont été plus performants que le groupe contrôle. Cependant, une mauvaise réalisation a été remarquée chez le groupe expérimental au niveau du voisement de la lettre $<\mathrm{s}>$ entre deux voyelles et à la liaison.

Tableau 5. Analyse du troisième test.

\begin{tabular}{llrrrrr}
\hline & Groupe & Nombre & \multicolumn{1}{c}{ Moyenne } & Ecart-type & \multicolumn{1}{c}{$t$} & \multicolumn{1}{c}{$P$} \\
\hline \multirow{2}{*}{ Test 3 } & expérimental & 42 & 82.50 & 9.587 & 2.020 & .047 \\
& contrôle & 40 & 78.25 & 9.451 & & \\
\hline
\end{tabular}

Le résultat du troisième test a confirmé les deux premières évaluations : la méthode syllabique permet aux étudiants une meilleure prononciation du français ainsi qu'une meilleure maîtrise de l'orthographe dans les évaluations identiques basées sur des critères au niveau de la prononciation, de l'intonation ainsi que de la fluidité des productions orales. Toutefois, nous constatons la même difficulté chez les deux groupes une mauvaise distinction de la réalisation de la lettre $<\mathrm{e}>$ sous différents contextes orthographique (e.g. «ce » prononcé $[\mathrm{s} \varepsilon]$ ou « merci » prononcé [mərsi]) etc.).

Tableau 6. Analyse du quatrième test.

\begin{tabular}{llrrrrr}
\hline & Groupe & \multicolumn{1}{c}{ Nombre } & \multicolumn{1}{c}{ Moyenne } & Ecart-type & \multicolumn{1}{c}{$t$} & \multicolumn{1}{c}{$P$} \\
\hline \multirow{2}{*}{ Test 4 } & expérimental & 42 & 84.12 & 10.381 & 3.159 & .002 \\
& contrôle & 40 & 76.40 & 11.729 & & \\
\hline
\end{tabular}

Les quatre tests ont prouvé que le groupe expérimental avait un taux de prononciation correcte plus élevé par rapport au groupe contrôle. Il est à noter que les étudiants du groupe API tendent à transcrire les mots qui leur sont moins familiers en alphabet phonétique. Même si les étudiants avaient mis des transcriptions phonétiques, nous avons constaté, pendant le test, des hésitations ou de l'incertitude chez les apprenants à cause d'oublis ou de confusions des signes alphabétiques. Plusieurs confusions ont été constatées entre les deux prononciations de la lettre $<\mathrm{c}>$ ([s] vs [k]) et en particulier $<\mathrm{g}>,<\mathrm{gu}>([3] \mathrm{vs}[\mathrm{g}])$. Entre autres, la réalisation des voyelles arrondies et la distinction de la lettre $<\mathrm{e}>$ constituent toujours la cause de l'hésitation et des erreurs pour nos étudiants lors de la prononciation.

En résumé, les résultats des tests suggèrent que la méthode syllabique aide les apprenants à mieux prononcer, et diminue leur charge de mémoire par rapport à la méthode API. La méthode syllabique améliore la compétence de prononciation globale de l'apprenant, mais il existe des points faibles en ce qui concerne des mots dont l'orthographe est opaque. Cette méthode mérite donc notre attention au niveau de l'enseignement de la prononciation du FLE en Chine. Cependant, il faut souligner que cette étude comparative ne consiste pas à opposer les deux approches de l'enseignement de la prononciation, il nous faut relever l'importance de l'interaction entre transparence de l'écrit et opacité de l'oral (Galazzi, 2017 : 293) et en même temps l'attention doit être accordée à l'irrégularité orthographique et aux éléments suprasegmentaux en vue d'une prononciation correcte, adéquate et réussie dans la communication orale. 


\section{Conclusion et perspective}

Certes, l'API joue un rôle important de médiation entre l'écrit et l'oral dans l'enseignement de la prononciation du français. Il connaît cependant plusieurs interférences avec les graphies françaises, la transcription phonétique anglaise ou le pinyin dans le contexte chinois. La méthode syllabique, pouvant combler les défauts de l'API, possède également ses limites. Car théoriquement, l'apprentissage de la prononciation d'une langue étrangère et l'apprentissage de la lecture de la langue maternelle ne sont pas identiques. L'approche syllabique a été conçue dans un premier temps pour enseigner la lecture aux enfants natifs, pour les amener à reconnaître la forme écrite de leur langue. Aussi présumet-elle une capacité à parler et à comprendre cette langue au préalable. Ce qui n'est pas le cas de l'apprentissage du FLE en Chine. Signalons également que la méthode syllabique s'avère moins efficace que l'API concernant la lecture des mots dont l'orthographe ne correspond pas à la prononciation, prenant comme exemple un nombre important de lettres muettes et le mot « oiseau » dont la réalisation phonétique /wazo/ ne correspond à aucune lettre orthographique! Etant donné que nombre d'éléments, comme les connaissances métalinguistiques, pourraient également influer sur le développement de la capacité procédurale de la prononciation, nous devons donc nous baser sur la méthode syllabique, mais compléter cette dernière par d'autres supports (orthographique, morphologique, sémantiques ou pragmatiques etc.) comme ressources supplémentaires du cours de phonétique française afin d'atteindre le but global qu'est la communication adéquate et réussie dans cette langue.

De toute manière, il serait judicieux d'intégrer également les éléments extralinguistiques comme la motivation, les stratégies ou l'ambiance d'apprentissage en vue d'une approche hétérogène propre à chaque situation de classe et à des profils d'apprenants différents (cf. Arnold, $2006: 413$ ) en ce qui concerne l'enseignement de la prononciation du français aujourd'hui. Au final, notre étude connaît ses limites : le suprasegmental n'a pas été traité à cause de la limite des heures d'enseignement et les données que nous avons recueillies ne sont pas exhaustives pour représenter tous les cas de l'apprentissage du français en Chine. Nos analyses permettent de commencer à interpréter l'importance et l'efficacité de la méthode syllabique au sein de l'enseignement universitaire du FLE en contexte chinois. Enfin, il faudra également avoir recours à d'autres sources pour nourrir et approfondir ces interprétations, esquissées seulement à partir d'un corpus assez réduit. Nous entamerons les réflexions, notamment à partir d'autres expériences empiriques, recherches-actions ainsi que d'entretiens plus approfondis auprès des enseignants et des étudiants dans les futures recherches.

Cette étude est financée par CSC scholarship $\mathrm{N}^{\circ} 201706380147$ et fait partie du projet 2016 niandu Guangdong sheng benke gaoxiao jiaoxue zhiliang yu jiaoxue gaige gongcheng xiangmu: "Hulianwang+" beijing xia jiyu fanzhuan ketang" moshi de gaoxiao xiaoyuzhong jiaoxue gaige yanjiu - yi « Daxue Fayu» kecheng weili. Nous remercions les enseignants et les étudiants qui ont participé aux enquêtes, et nos remerciements vont également au professeur Guy Achard-Bayle, au professeur Zhihong Pu ainsi qu'aux relecteurs du comité scientifique qui ont relu le présent travail en y apportant de nombreuses améliorations. 


\section{Références bibliographiques}

Arnold, J. (2006). Comment les facteurs affectifs influencent-ils l'apprentissage d'une langue étrangère ? Études de linguistique appliquée, 407-425.

Catach, N. (2011). L'orthographe (10 édition). Paris : Puf.

Cheng, M.-S. \& Fan, W.-F. (2011). Linking the Sounds with the Spelling in Learning English Words: Using Natural Pronunciation Symbols. Foreign Languages in China, 76-81.

Colé, P. \& Fayol, M. (2000). Reconnaissance de mots écrits et apprentissage de la lecture : rôle des connaissances morphologiques. L'acquisition du langage, 151-181.

Conseil de l'Europe. (2001). Cadre européen commun de référence pour les langues : apprendre, enseigner, évaluer. Paris : Didier.

Cuq, J.-P. \& Gruca, I. (2005). Cours de didactique du français, langue étrangère et seconde. Grenoble : Presses universitaires de Grenoble.

Detey, S., Fontan, L. \& Pellegrini, T. (2016). Traitement de la prononciation en langue étrangère : approches didactiques, méthodes automatiques et enjeux pour l'apprentissage. Revue Traitement Automatique des Langues, 15-39.

Durant, J. (2005). La phonétique classique : l'Association Phonétique Internationale et son alphabet. In Nguyen, N., Wauquier-Gravelines, S. \& Durand, J. (dir.). Phonologie et phonétique : forme et substance, Paris : Hermès Science.

Galazzi, E. (2017). Variations phonétiques contemporaines: transparence et opacité dans le marché des langues globalisé du côté du FLE. In Tyne H., Bilger, M., Cappeau, P. \& Guerin P. (dir.). La variation en question(s). Bruxelles : Peter Lang.

Gougenheim, G. (1935). Eléments de phonologie française. Paris : Les Belles-Lettres.

Iivonen, A. \& Nevalainen, T. (1998). Vieraan kielen fonetiikan opetuksen lähtökohtia. Helsinki : University of Helsinki.

International Phonetic Association. (1949). The Principles of the International Phonetic Association. London : International Phonetic Association.

Jaffré, J. (2003). La linguistique et la lecture-écriture : de la conscience phonologique à la variable « orthographe ». Revue des sciences de l'éducation, 37-49.

Kalmbach, J. M. (2011). Phonétique et prononciation du français pour apprenants finnophones. Jyväskylän : Jyväskylän yliopisto.

Krashen, S. (1982). Principles and practice in Second Language Acquisition. Oxford : Pergamon.

Lauret, B. (2007). Enseigner la prononciation du français : questions et outils. Paris : Hachette.

Ma Q.-W. \& Zhao Y.-G. (2017). Phonology, Phonetics and Pronunciation Teaching. Journal of Beijing International Studies University, 40-55.

Nicot-Guillorel, M. (2012). Bilinguisme et apprentissage de la lecture à Madagascar : quelle place pour la phonologie? Revue française de pédagogie, 97-114.

Pierret, J.-M. (1981). Phonétique du français. Notions de phonétique générale et phonétique historique du français. Série Pédagogique de l'Institut de Linguistique de Louvain, 1-245.

Robert, J.-M. (2004). Proximité linguistique et pédagogie des langues non maternelles. Études de linguistique appliquée, 499-511.

Snider, V.-E. (1997). The Relationship Between Phonemic Awareness and Later Reading Achievement. The Journal of Educational Research, 203-211.

Troubetzkoy, N.-S. (1986). Principes de phonologie. Paris, Klincksieck. 
Woore, R. (2011). Investigating and developing beginner learners' decoding proficiency in second language French: an evaluation of two programmes of instruction. Thèse de doctorat, University of Oxford.

Zhongguo Guojia Biaozhunhua Guanli Weiyuanhui. (2012). Basic rules of the Chinese phonetic alphabet orthography (GB/T 16159-2012). Pékin : Ministry of Education of the People's Republic of China.

Zhongguo Wenzi Gaige Weiyuanhui. (1958). Hanyu Pinyin Fang'an. Pékin : Ministry of Education of the People's Republic of China.

Ziegler, J.-C. \& Montant, M. (2005). Le développement de la lecture dans différentes langues : un problème de taille. Langage et l'homme, 149-157. 